\title{
Heat Treatment of Cast Magnetite Electrode*
}

\author{
Takashi NAgAI, Hironobu TATENO and Takeshi TAKEI**
}

Although cast magnetite is widely used as insoluble anode in the electrolytic production of chlorate, it is not good enough in electrical conductivity, corrosion resistivity and mechanical strength. In order to improve it, the authors studied on the effect of its heat treatment applied to it.

As preliminary observations, we examined the microstructure of the magnetite, and it was found that there were two types of microstructure according to the casting condition as shown in Fig. 1 and 2 , that is, one constisted of parallel growth of the $\left[\mathrm{Fe}_{3} \mathrm{O}_{4}\right]$ crystal and the other of random crystal aggregates.

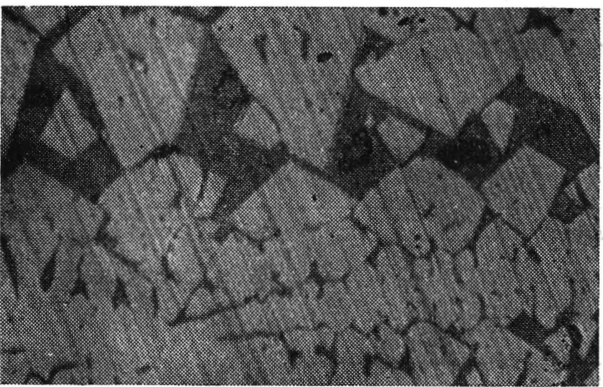

Fig. 1 Parallel growth of $\left[\mathrm{Fe}_{3} \mathrm{O}_{4}\right]$ crystal.

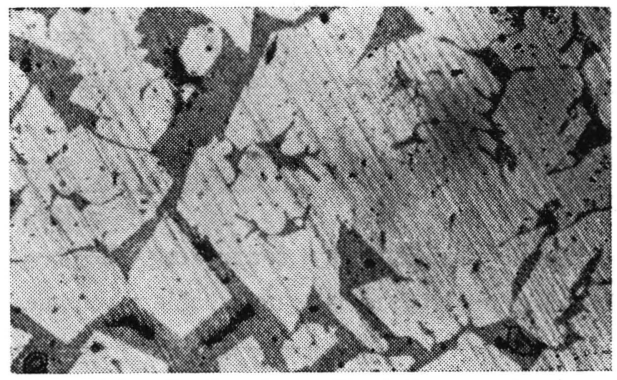

Fig. 2 Random $\left[\mathrm{Fe}_{3} \mathrm{O}_{4}\right]$ crystal aggregates.

Conventionally, we defined the direction of parallel crystal growth as $x$-direction in the case of orientated crystal arrangement, and took $y$-direction perpendicular to $x$-direction.

The specific electrical resistance at $x$-direction is much lower than that of $y$-direction as shown in Table 1. Mechanical strength at $x$-direction is also lower than that of the other. But there is no distinct difference in the corrosion resistivity between the planes of different directions.

Table 1 Relation between the Arrangement of $\left[\mathrm{Fe}_{3} \mathrm{O}_{4}\right]$ Crystals and the Specific Resistance

\begin{tabular}{|c|c|c|c|}
\hline \multirow{2}{*}{$\begin{array}{c}\text { Crystal } \\
\text { Arrangement }\end{array}$} & \multicolumn{2}{|c|}{ Specific Resistance $(\Omega-\mathrm{cm})$} & \multirow{2}{*}{$\begin{array}{l}\text { Mechanical } \\
\text { Strength }\end{array}$} \\
\hline & $x$-direction & $y$-direction & \\
\hline regular & 0.2 & 7.0 & week \\
\hline random & 0.1 & 0.2 & strong \\
\hline locally regular & 0.5 & 5.0 & medium \\
\hline
\end{tabular}

Heat treatments were applied to the test pieces cut out from the commercial magnetite electrode, at temperatures between $900^{\circ}$ and $1200^{\circ} \mathrm{C}$ for 5 minutes, and as shown in Table 2 and 3 , it was found that, in any cases, and in any directions, the electrical resistance evidently decreased by the heat treatment. The decrease is sometimes very remarkable. The change of corrosion resistivity was scarcely found by the heat treatment.

Experimental magnetite electrodes were prepared by melting commercial electrode and by casting it into graphite mold. The pieces were cut out from them, and heat treatment was applied at several temperatures. In this case, the electrical resistance at any directions increased at first, and then

* Fundamental Studies on the Electrolytic Production of Chlorate (Part 6)

** Faculty of Engineering, Keiogijuku University, Koganei-City, Tokyo

The original written in Japanese can be seen in J. Electrochem. Soc. Japan, 27, 381 (1959). 
Table 2 The Effect of Heat Treatment on the Specific Resistance of Commercial Magnetite Electrode ( $x$-direction)

\begin{tabular}{c|c|c|c}
\hline \hline \multirow{2}{*}{$\begin{array}{c}\text { Temperature } \\
\left({ }^{\circ} \mathrm{C}\right)\end{array}$} & \multicolumn{2}{|c|}{ Specific Resistance $(\Omega-\mathrm{cm})$} & $\left(R / R_{0}\right) \times 100$ \\
\cline { 2 - 4 } & $\begin{array}{c}\text { Before Heat Treatment } \\
\left(R_{0}\right)\end{array}$ & $\begin{array}{c}\text { After Heat Treatment } \\
(R)\end{array}$ & \\
\hline 800 & 0.129 & 0.116 & 90 \\
900 & 0.117 & 0.089 & 76 \\
1000 & 0.132 & 0.073 & 55 \\
1100 & 0.071 & 0.037 & 52 \\
1200 & 0.107 & 0.060 & 56 \\
\hline
\end{tabular}

(Heat treatment : 5 mins)

Table 3 The Effect of Heat Treatment on the Specific Registance of Commercial Magnetite Electrode ( $y$-direction)

\begin{tabular}{|c|c|c|c|}
\hline \multirow{2}{*}{$\begin{array}{l}\text { Temperature } \\
\left({ }^{\circ} \mathrm{C}\right)\end{array}$} & \multicolumn{2}{|c|}{ Specific Resistance $(\Omega-\mathrm{cm})$} & \multirow{2}{*}{$\left(R / R_{0}\right) \times 100$} \\
\hline & $\underset{\left(R_{0}\right)}{\text { Before Heat Treatment }}$ & $\underset{(R)}{\text { After Heat Treatment }}$ & \\
\hline 900 & 0.306 & 0.139 & 45 \\
\hline 1100 & 0.283 & 0.136 & 48 \\
\hline 1200 & 0.316 & 0.100 & 31 \\
\hline
\end{tabular}

(Heat treatment : 5 mins)

decreased gradually by continuing the heat treatment as shown in Fig. 3 . This first increase in electrical resistance is a noticeable phenomenon.

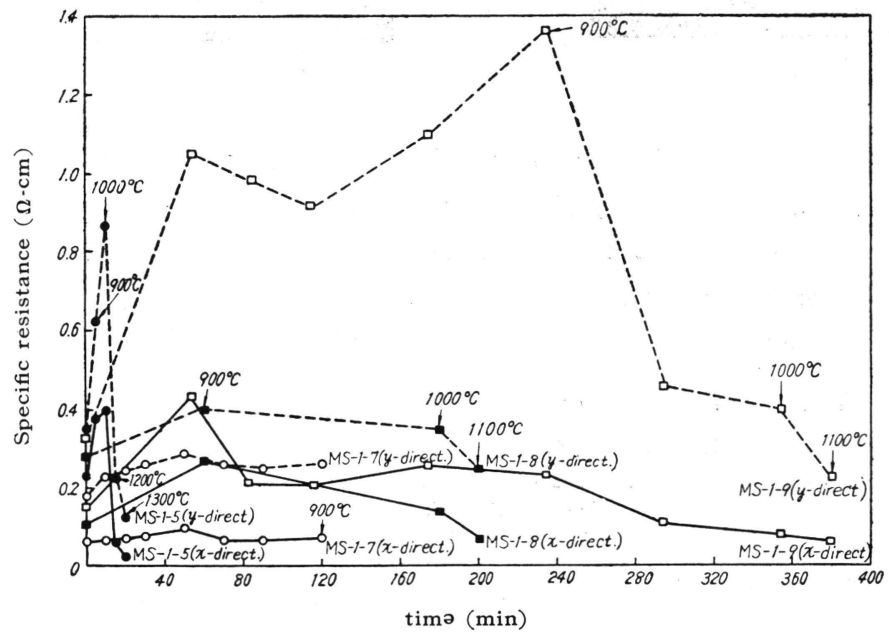

Fig. 3 Heat treatment of experimental electrodes.

To consider the cause of effect of heat treatment, several special experiments were carried out and the following results were obtained.

(1) Even in the heat treatment between the temperatures $400^{\circ}$ and $900^{\circ} \mathrm{C}$, an increase in electrical resistance was observable in the case of experimental electrode.

(2) Even in the case of hollow cylindrical experimental electrode, the increase in electrical resistance was observable.

(3) Even in the case of experimental electrode consisted of relatively large $\left[\mathrm{Fe}_{3} \mathrm{O}_{4}\right]$ crystals, the increase of electrical resistance was also observable.

(4) In the case of experimental electrode slowly cooled after casting, the increase in electrical resistance was not observable any more.

(5) Electrical resistance at bottom (closed) end of the commercial electrode increased at first just 


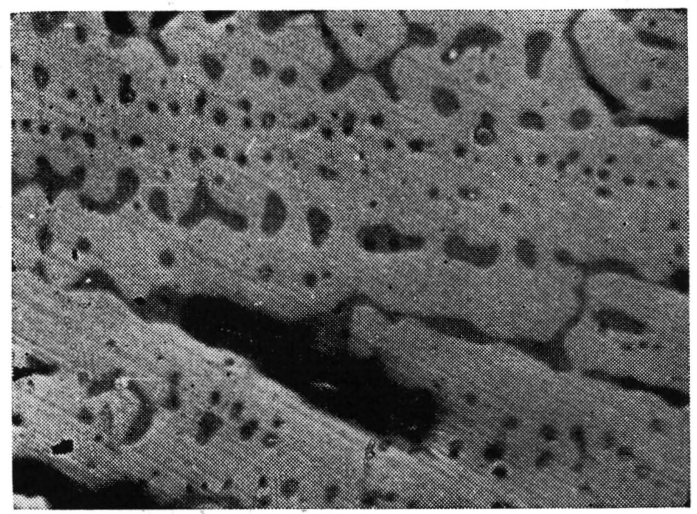

Fig. 4 The crystal configuration of magnetite electrode heat treated at $1200^{\circ} \mathrm{C}$ for 5 mins.

like as in the experimental electrode.

A marked change of microstructure was observable by the heat treatment at $1200^{\circ} \mathrm{C}$ as shown in Fig. 4, though the change was very slight in the case of heat treatment at $900^{\circ} \mathrm{C}$. Chemical composition did not change by the heat treatments.

From these experimental results and from X-ray analysis, we could explain the reason of the change of the electrical resistance by heat treatments to some extents as follows.

(1) The increase of electrical resistance by heat treatment in the case of experimental electrode is considered to be due to the inherent increase of electrical resistance $\left[\mathrm{Fe}_{3} \mathrm{O}_{4}\right]$ crystal by some change, for example, precipitation of secondary phase.

(2) The decrease of electrical resistance by further heat treatment at comparatively lower temperature in both commercial and experimental electrode is also due to the inherent decrease of electrical resistance of $\left[\mathrm{Fe}_{3} \mathrm{O}_{4}\right]$ crystal by just contrary change as (1) for example, dissolution of secondary phase.

(3) The decrease of electrical resistance by heat treatment at high temperatures may be caused by both the same reason as (2) and by the change of crystal configuration.

(Received September 29, 1958) 